\title{
Continuous pseudo-hairy spaces and continuous pseudo-fans
}

\author{
by
}

\author{
Janusz R. Prajs (Opole and Pocatello, ID)
}

\begin{abstract}
A compact metric space $\widetilde{X}$ is said to be a continuous pseudo-hairy space over a compact space $X \subset \widetilde{X}$ provided there exists an open, monotone retraction $r$ : $\widetilde{X} \stackrel{\text { onto }}{\longrightarrow} X$ such that all fibers $r^{-1}(x)$ are pseudo-arcs and any continuum in $\widetilde{X}$ joining two different fibers of $r$ intersects $X$. A continuum $Y_{X}$ is called a continuous pseudo-fan of a compactum $X$ if there are a point $c \in Y_{X}$ and a family $\mathcal{F}$ of pseudo-arcs such that $\bigcup \mathcal{F}=Y_{X}$, any subcontinuum of $Y_{X}$ intersecting two different elements of $\mathcal{F}$ contains $c$, and $\mathcal{F}$ is homeomorphic to $X$ (with respect to the Hausdorff metric). It is proved that for each compact metric space $X$ there exist a continuous pseudo-hairy space over $X$ and a continuous pseudo-fan of $X$.
\end{abstract}

Investigation of pseudo-arcs started from the construction [5] of Knaster's hereditarily indecomposable continuum in 1922. In fact, being chainable, Knaster's continuum is a pseudo-arc [2]. In the sense of Baire category pseudo-arcs are known to be the most common compact connected spaces, contained in: $n$-manifolds $(n>1)$, the Hilbert cube, the Menger curve, its higher dimensional analogues etc. They could be compared to the irrationals among the real numbers. Therefore, extraordinary properties of the pseudo-arc could be expected throughout the topology of separable metric spaces. However, most of known results on the pseudo-arc deal either with its internal properties, or with its position in the plane.

Among the results concerning the pseudo-arc there is the Bing-Jones construction [3] of the circle of pseudo-arcs, i.e. of a curve admitting an open mapping onto a circle with terminal pseudo-arcs as all fibers. Later Lewis [8] generalized this construction so that the circle is replaced by any 1-dimensional continuum, and asked whether all curves admitting a continuous decomposition into pseudo-arcs with a fixed curve as quotient are

2000 Mathematics Subject Classification: Primary 54F15; Secondary 54G99, 54F50.

Key words and phrases: compact metric space, continuous decomposition of compactum, pseudo-arc, pseudo-hairy space, pseudo-fan. 
topologically unique. In a previous paper [9] the author answered this question in the negative constructing a selectible circle of pseudo-arcs, i.e. a curve admitting an open monotone retraction onto a circle $\mathcal{S}$ with pseudo-arcs as all fibers, such that no two fibers are joined by a continuum outside of $\mathcal{S}$. In the same paper this result was extended to any curve instead of the circle.

Dimension 1 is an essential limitation in the constructions of Bing-JonesLewis, if terminality of the fibers is to be preserved. Indeed, any continuum admitting a continuous decomposition into terminal nondegenerate curves must be 1-dimensional. If these curves are tree-like, the dimension of the quotient cannot be greater by Theorem 8, p. 136 of [4].

The selectible curves of pseudo-arcs from [9] have, in a sense, "almost terminal" fibers, i.e. they behave like terminal continua everywhere outside of the distinguished selector. Therefore any extension of the results of [9] to higher dimensional spaces seemed rather unexpected to the author. Nevertheless, in this paper we show that for any metric compactum $X$ (actually, for any separable metric space - see Remark 2) there exists a space $\widetilde{X}$ admitting an open monotone retraction $r$ onto $X$ with pseudo-arcs as all fibers $r^{-1}(x)$, such that no two of them can be joined with a continuum outside of $X$. Since the constructed spaces need not be curves any more, we call $\widetilde{X}$ a continuous pseudo-hairy space over $X$.

Though being fibers of such constructions seems to be very rare among continua, the continuous pseudo-hairy space over the pseudo-arc (and its further iterations) occur to have this property (comp. a comment in the final part of [9]).

When we shrink the set $X$ to a point in the pseudo-hairy space $\tilde{X}$, a tree-like (or more precisely, star-like, see Proposition 18) curve is obtained. It is a one-point union of a compact family $\mathcal{F}$ of pseudo-arcs (a "bouquet" of pseudo-arcs) such that $\mathcal{F}$ is homeomorphic to $X$. We call it a continuous pseudo-fan of $X$, and the distinguished point joining the pseudo-arcs is called the vertex of the pseudo-fan. By the results of this paper any metric compactum (of any dimension) has such a representation (see Theorem 16) in the form of a star-like curve. So, these representations are embeddable in $\mathbb{R}^{3}$ (where $\mathbb{R}$ is the real line). Thus we obtain, in a sense, a reduction of the "world" of all metric compacta to the "world" of some special star-like curves in $\mathbb{R}^{3}$. Though we can represent higher dimensional compacta in terms of subsets of $\mathbb{R}$ even (continuous images of the Cantor set, or geometrically, encoding with sequences of $0-1$ numbers), our representation seems to be more intuitive because we can "see" the space $X$ in the topological structure of its continuous pseudo-fan. Namely, $\mathcal{F}$ (homeomorphic to $X$ ) is the family of all maximal pseudo-arcs, or equivalently, of all maximal irreducible subcontinua with the vertex as a point of irreducibility. 
The continuous pseudo-fans can be represented in the form of an inverse $\operatorname{limit} \lim \left(X_{n}, f_{n}\right)$, where $X_{n}$ 's are $n$-ods and $f_{n}$ 's are piecewise linear, light mappings preserving the vertices of $X_{n}$ 's. The proof of this is existential only, so we have the following problem.

Problem 1. Find a method to represent the continuous pseudo-fans of particular spaces (say: an arc, an n-cube, the Menger curve, the Hilbert cube, etc.) in the form of the inverse limit of n-ods with piecewise linear bonding mappings preserving the vertices.

This paper is a continuation of an earlier study [9]. Here we apply some essential tool proved in that paper (Th. 3 of [9]), and use some new ones to construct a special decomposition of the product of the unit segment and the infinite-dimensional torus $\mathcal{S}^{\omega}$ into pseudo-arcs so that the continuous pseudo-hairy space over this torus can be derived from the decomposition. The methods developed may have other applications (see Remark 1). The main result follows by the universality of $\mathcal{S}^{\omega}$ among all metric compacta. In the last section we collect some basic observations on pseudo-hairy spaces and pseudo-fans.

Preliminaries. Only metric spaces are considered. For any space $X$ the space of all nonempty continua in $X$ with the Hausdorff metric $d_{\mathrm{H}}$ is denoted by $C(X)$. The symbol Lim stands for the limit of closed sets with respect to the metric $d_{\mathrm{H}}$. Mappings are assumed to be continuous.

For any compact space $X$ define $\xi(X)=\sup \left\{\min \left\{d_{\mathrm{H}}(K, K \cup L), d_{\mathrm{H}}(L, K \cup L)\right\}: K, L \in C(X)\right.$ and $\left.K \cap L \neq \emptyset\right\}$. Observe that if $\varepsilon>\xi(X)$, then $X$ is $\varepsilon$-hereditarily indecomposable in the sense of [9], and $X$ is hereditarily indecomposable if and only if $\xi(X)=0$.

A compact space $\widetilde{X}$ is said to be a pseudo-hairy space over $X \subset \widetilde{X}$ if there is a retraction $r: \widetilde{X} \rightarrow X$ such that each fiber $r^{-1}(x)$ is a pseudo-arc, and each continuum intersecting two different fibers $r^{-1}(x), r^{-1}(y)$ intersects $X$. Such a set $X$ is then called the base of the pseudo-hairy space $\widetilde{X}$. If, additionally, $r$ is an open mapping we say that $\widetilde{X}$ is a continuous pseudohairy space over $X$.

A continuum $Y$ is said to be a pseudo-fan provided there are a point $c \in Y$ (called the vertex of $Y$ ) and an upper semicontinuous family $\mathcal{F}$ of pseudo-arcs in $Y$ such that $\bigcup \mathcal{F}=Y$ and any continuum in $Y$ joining two different elements of $\mathcal{F}$ contains $c$. Observe that then $P_{1} \cap P_{2}=\{c\}$ for any two different pseudo-arcs $P_{1}, P_{2}$ in $\mathcal{F}$. If, additionally, the family $\mathcal{F}$ is homeomorphic (in the sense of the Hausdorff distance) to some compactum $X$, we say that $Y$ is a continuous pseudo-fan of $X$. Let $f: X \rightarrow Y$ be a surjective mapping, and let $\mathcal{D}_{X}, \mathcal{D}_{Y}$ be decompositions of the spaces $X$ 
and $Y$, respectively. We say that $f$ transforms $\mathcal{D}_{X}$ onto $\mathcal{D}_{Y}$ provided $\mathcal{D}_{X}=$ $\left\{f^{-1}(D): D \in \mathcal{D}_{Y}\right\}$. For any mapping $f: X \rightarrow Y$ with $X \subset Y$ we define

$$
d(f)=\sup \{d(x, f(x)): x \in X\},
$$

and if $d(f)<\varepsilon$, we say that $f$ is an $\varepsilon$-pushing. A family of mappings $f_{\alpha}$ : $X_{\alpha} \rightarrow Y_{\alpha}$ is said to be uniformly equicontinuous provided for any $\varepsilon>0$ there is a $\delta>0$ such that $d\left(f_{\alpha}(x), f_{\alpha}(y)\right)<\varepsilon$ for each $\alpha$ and each pair $x, y \in X_{\alpha}$ with $d(x, y)<\delta$.

A finite family of sets is called an $\varepsilon$-chain (resp. $\varepsilon$-star cover) provided its elements have diameters less than $\varepsilon$ and its nerve is an arc (resp. a finite, connected, acyclic graph with at most one ramification point).

For a sequence $x_{n}$ of real numbers we use the following standard notation: $\limsup x_{n}=\lim _{n} \sup \left\{x_{m}: m>n\right\}, \liminf x_{n}=\lim _{n} \inf \left\{x_{m}: m>n\right\}$.

Inverse sequences converging in complete metric spaces. In this section basic tools are prepared to prove the main result of the paper. They may also have other applications (see Remark 1).

Let $K_{n}$ be a sequence of closed subsets of a metric space $X$ and let $f_{n}: K_{n+1} \rightarrow K_{n}$ be mappings. We say that the inverse sequence $\left(K_{n}, f_{n}\right)$ converges in the space $X$ provided that:

(i) the $\operatorname{limits} \operatorname{Lim} K_{n} \subset X$ and $\lim x_{n}$ in $X$ exist for each $\left(x_{1}, x_{2}, \ldots\right) \in$ $\lim \left(K_{n}, f_{n}\right)$, and the function $h\left(x_{1}, x_{2}, \ldots\right)=\lim x_{n}$ is a homeomorphism between $\lim \left(K_{n}, f_{n}\right)$ and $\operatorname{Lim} K_{n}$; and

(ii) identifying $\lim \left(K_{n}, f_{n}\right)$ and $\operatorname{Lim} K_{n}$ by the homeomorphism $h\left(x_{1}, x_{2}, \ldots\right)=\lim x_{n}$, the projections of the inverse limit uniformly converge to the identity on $\operatorname{Lim} K_{n}$.

The following result provides a sufficient condition for the convergence of an inverse sequence in a complete metric space. Formulated in other terms, this theorem was proved in [1] (Th. I) for compact spaces. A closely related idea was applied in [9] (Th. 4). We omit the proof.

TheOREM 1. Let $X$ be a complete metric space and $f_{n}: K_{n+1} \rightarrow K_{n}$ be a sequence of surjective mappings for closed subsets $K_{n}$ of $X$ such that:

(a) for each $\varepsilon>0$ there exists an integer $i>0$ such that $\operatorname{diam}\left\{x_{i}, x_{i+1}, \ldots\right\}$ $<\varepsilon$ for every thread $\left(x_{1}, x_{2}, \ldots\right) \in \lim \left(K_{n}, f_{n}\right)$; and

(b) the mappings $f_{i} \circ f_{i+1} \circ \ldots \circ f_{i+j}$ for all $i, j$ are uniformly equicontinuous.

Then the inverse sequence $\left(K_{n}, f_{n}\right)$ converges in $X$.

We sketch a general inductive procedure which leads to a sequence of mappings satisfying conditions (a) and (b) of Theorem 1 . The procedure implicitly appears in [1] (Lemmas 1, 2 and Th. III), and in [9] (the proof 
of Th. 5). Now we extract it for convenience with a view to possible future applications.

A procedure of constructing inverse sequences converging in complete metric spaces. Let $X$ be a complete metric space. There is an inductive procedure of choice of closed sets $K_{n}$ in $X$ and surjective mappings $f_{n}$ : $K_{n+1} \rightarrow K_{n}$ which satisfy condition (*) below; the procedure leads to the inverse sequence $\left(K_{n}, f_{n}\right)$ which converges in $X$.

At any inductive step, when sets $K_{1}, \ldots, K_{n}$ and mappings $f_{1}, \ldots$ $\ldots, f_{n-1}$ are already fixed, there is an $\varepsilon_{n}>0$ such that any closed set $K \subset X$ with a uniformly continuous surjective mapping $f: K \rightarrow K_{n}$ satisfying $d(f)<\varepsilon_{n}$ can be taken for $K_{n+1}$, and $f$ can be taken for $f_{n}$.

Indeed, fix a uniformly continuous surjection $f_{1}: K_{2} \rightarrow K_{1}$.

For any integer $n>0$ and any mapping $f: A \rightarrow B$ define $\delta(f, n)=$ $\inf (\{1 / n\} \cup\{d(x, y): x, y \in A$ and $d(f(x), f(y)) \geq 1 / n\})$. Observe that a family of functions $f_{\alpha}$ is uniformly equicontinuous if and only if for each $n$,

$$
\inf _{\alpha} \delta\left(f_{\alpha}, n\right)>0
$$

Let $\varepsilon_{1}=1$. Assume mappings $f_{1}, \ldots, f_{n-1}$ and numbers $\varepsilon_{1}, \ldots, \varepsilon_{n-1}$ are already defined. Define $g_{i j}=f_{i} \circ f_{i-1} \circ \ldots \circ f_{j-1}: K_{j} \rightarrow K_{i}$ for $i<j$. Let

$$
\varepsilon_{n}=\min \left(\left\{\varepsilon_{n-1} / 2\right\} \cup\left\{\frac{1}{5} \delta\left(g_{i j}, n\right): i<j \leq n\right\}\right) .
$$

Then we choose any closed set $K_{n+1} \subset X$ with a surjective uniformly continuous mapping $f_{n}: K_{n+1} \rightarrow K_{n}$ satisfying $d\left(f_{n}\right)<\varepsilon_{n}$. This completes the construction.

One can prove that $\delta\left(g_{i j}, n\right) \geq \varepsilon_{n}$ for all $i, j, n$ with $i<j$. This implies the uniform equicontinuity of all mappings $g_{i j}$, and thus (b) of Theorem 1 is satisfied. Condition (a) follows from $\varepsilon_{n+1} \leq \varepsilon_{n} / 2$ for each $n$. Hence the inverse sequence $\left(K_{n}, f_{n}\right)$ converges in $X$ by Theorem 1 .

Let $X$ be a complete metric space, and $\Phi$ be a family of uniformly continuous surjective mappings between closed subsets of $X$ such that

(A) if an inverse sequence $\left(K_{n}, f_{n}\right)$ converges in $X$ and $f_{n} \in \Phi$, then the projections $p_{n}: \operatorname{Lim} K_{n}=\underset{\lim }{\longleftarrow}\left(K_{n}, f_{n}\right) \rightarrow K_{n}$ belong to $\Phi$.

For any two closed sets $K, L \subset X$ define

$$
\sigma(K, L)=\inf (\{\operatorname{diam} X\} \cup\{d(f): f \in \Phi \text { and } f \text { maps } K \text { onto } L\}) \text {. }
$$

Note that $\sigma$ need not be symmetric, and possibly $\sigma(K, L)=\infty$.

Define the following two convergences for closed sets in $X$.

We write $\lim _{\sigma} K_{n}=K$ provided $\lim \sigma\left(K_{n}, K\right)=0$, and $\operatorname{invlim}_{\sigma} K_{n}=K$ provided $\lim \sigma\left(K, K_{n}\right)=0$. The symbol invlim $\sigma$ should be distinguished from $\mathrm{lim}_{\longleftarrow}$ denoting the usual inverse limit. 
Let $\mathcal{G}$ be a family of closed subsets of $X$ and let $\psi$ be a real function on $\mathcal{G}$. Consider the following kind of semicontinuity of $\psi$ on $\mathcal{G}$ :

(B) if $\operatorname{invlim}_{\sigma} K_{n}=K$, then $\liminf \psi\left(K_{n}\right) \geq \psi(K)$;

and the following property:

(C) for any $K \in \mathcal{G}$ there exists a sequence $K_{n} \in \mathcal{G}$ such that $\lim _{\sigma} K_{n}$ $=K$ and $\lim \psi\left(K_{n}\right)=0$.

The proof of the following is straightforward.

EXAMPLE 2. For any family $\mathcal{G}$ of compact sets the function $\xi$ (defined in the Preliminaries) satisfies condition (B).

THEOREM 3. Let $\mathcal{G}$ be a family of closed sets in a complete metric space $X$ such that $\mathcal{G}$ is closed with respect to the $\operatorname{invlim}_{\sigma}$ convergence, let $\psi_{m}$ be a sequence of nonnegative functions on $\mathcal{G}$, and let $\mathcal{G}_{0} \subset \mathcal{G}$. If condition (B) is satisfied for each $\psi_{m}$ and condition $(\mathrm{C})$ is satisfied in $\mathcal{G}_{0}$ for each $\psi_{m}$, then for each $K \in \mathcal{G}_{0}$ there is a sequence $K_{n} \in \mathcal{G}$ such that $\lim _{\sigma} K_{n}=K$ and $\psi_{m}\left(K_{n}\right)=0$ for each $m, n$.

Proof. Let $m_{i}$ be a sequence in which each positive integer appears infinitely many times. Let $n$ be a positive integer and $K \in \mathcal{G}_{0}$. Define $L_{1, n}=K$. Applying the inductive procedure described after Theorem 1 and property (C) we find a sequence $L_{n, i}$ of members of $\mathcal{G}_{0}$ and a sequence of surjections $f_{n, i}: L_{n, i+1} \rightarrow L_{n, i}$ such that $\sum_{i=1}^{\infty} d\left(f_{n, i}\right)<1 / n$, the inverse sequence $\left(f_{n, i}, L_{n, i}\right)$ converges in $X$ with respect to $i$, and $\lim _{i} \psi_{m_{i}}\left(L_{n, i}\right)=0$. Namely, at each iductive step we additionally assume that $d\left(f_{n, i}\right)<1 /\left(n 2^{i+1}\right)$ and $\psi_{m_{i}}\left(L_{n, i}\right)<1 / i$. Let $K_{n}=\lim _{i}\left(f_{n, i}, L_{n, i}\right)$, and let $f_{n}: K_{n} \rightarrow K$ be the projection onto the first coordinate with respect to this inverse limit. Let $m>0$ be an integer. Using the projections of the inverse limit to the sets $L_{n, i}$ satisfying $m_{i}=m$ and applying (B) to the mapping $\psi_{m}$, we see that $\psi_{m}\left(K_{n}\right)=0$. Thus $\psi_{m}\left(K_{n}\right)=0$ for each $m$. Applying this argument for each $n$ we have $\lim _{\sigma} K_{n}=K$.

Now we introduce similar concepts for families of compact sets that form continuous (or upper semicontinuous) decompositions of a complete metric space. We leave the same symbols to lay stress on the similarities of these two approaches.

The following general observation is a consequence of the definition of the convergence of an inverse sequence. Notice that, though in this observation the mapping $f_{n}$, for any $n$, need not be $1-1$, according to the definition it induces a 1-1 function between the collections $\mathcal{D}_{n+1}$ and $\mathcal{D}_{n}$.

OBSERVATION 4. Let $\mathcal{D}_{n}$ be a sequence of continuous (resp. upper semicontinuous) decompositions of a complete metric space $X$ into compact sets and let $f_{n}: X \rightarrow X$ be surjective mappings such that $f_{n}$ transforms $\mathcal{D}_{n+1}$ 
onto $\mathcal{D}_{n}$. Suppose that the inverse sequence $\left(X_{n}, f_{n}\right)$, where $X_{n}=X$ for each $n$, converges in $X$, and let $D_{i} \in \mathcal{D}_{i}$ for some fixed $i$. Define inductively $D_{n-1}=f_{n-1}\left(D_{n}\right)$ for $n \leq i$, and $D_{n+1}=f_{n}^{-1}\left(D_{n}\right)$ for $n \geq i$. Then the inverse sequence $\left(D_{n}, g_{n}\right)$, where $g_{n}=f_{n} \mid D_{n+1}$, converges in $X$. The family of limits $\operatorname{Lim} D_{n}$, for all sequences $\left\{D_{n}\right\}$ defined as above, forms a continuous (resp. upper semicontinuous) decomposition of $X$.

Fix a family $\Phi$ of uniformly continuous surjective mappings $f: X \rightarrow X$ which satisfies condition (A) for $K_{n}=X$. If $\mathcal{D}_{1}, \mathcal{D}_{2}$ are two continuous (or upper semicontinuous) decompositions of a complete metric space $X$, define (1) $\sigma\left(\mathcal{D}_{1}, \mathcal{D}_{2}\right)=\inf \left(\{\operatorname{diam} X\} \cup\left\{d(f): f \in \Phi\right.\right.$ transforms $\mathcal{D}_{1}$ onto $\left.\left.\mathcal{D}_{2}\right\}\right)$.

Note that $\sigma$ need not be symmetric, and possibly $\sigma\left(\mathcal{D}_{1}, \mathcal{D}_{2}\right)=\infty$.

Define the following two convergences for continuous (or upper semicontinuous) decompositions of $X$.

We write $\lim _{\sigma} \mathcal{D}_{n}=\mathcal{D}$ provided $\lim \sigma\left(\mathcal{D}_{n}, \mathcal{D}\right)=0$, and $\operatorname{invlim}_{\sigma} \mathcal{D}_{n}=\mathcal{D}$ provided $\lim \sigma\left(\mathcal{D}, \mathcal{D}_{n}\right)=0$. As in the previous case, the symbol invlim $\sigma$ should not be confused with $\varliminf_{\text {im. }}$.

Let $\widehat{\mathcal{G}}$ be a set of continuous (or upper semicontinuous) decompositions of a complete metric space $X$ into compact subsets, and let $\psi$ be a real function on $\widehat{\mathcal{G}}$. Consider the following kind of semicontinuity of $\psi$ on $\widehat{\mathcal{G}}$ :

$$
\text { if } \operatorname{invlim}_{\sigma} \mathcal{D}_{n}=\mathcal{D} \text {, then } \lim \inf \psi\left(\mathcal{D}_{n}\right) \geq \psi(\mathcal{D}) ;
$$

and the following property:

(C1) for any $\mathcal{D} \in \widehat{\mathcal{G}}$ there exists a sequence $\mathcal{D}_{n} \in \widehat{\mathcal{G}}$ such that $\lim _{\sigma} \mathcal{D}_{n}=$ $\mathcal{D}$ and $\lim \psi\left(\mathcal{D}_{n}\right)=0$.

The proof of the following theorem uses Observation 4. The argument is similar to the one of Theorem 3, so we omit it.

THEOREM 5. Let $\widehat{\mathcal{G}}$ be a set of continuous (or upper semicontinuous) decompositions of a complete metric space $X$ into compact subsets such that $\widehat{\mathcal{G}}$ is closed with respect to the invlim $_{\sigma}$ convergence, let $\psi_{m}$ be a sequence of nonnegative functions on $\widehat{\mathcal{G}}$, and let $\widehat{\mathcal{G}}_{0} \subset \widehat{\mathcal{G}}$. If condition (B1) is satisfied for each $\psi_{m}$ and condition (C1) is satisfied in $\widehat{\mathcal{G}}_{0}$ for each $\psi_{m}$, then for each $\mathcal{D} \in \widehat{\mathcal{G}_{0}}$ there is a sequence $\mathcal{D}_{n} \in \widehat{\mathcal{G}}$ such that $\lim _{\sigma} \mathcal{D}_{n}=\mathcal{D}$ and $\psi_{m}\left(\mathcal{D}_{n}\right)=0$ for each $m, n$.

REMARK 1. Many well known and extensively studied topological properties of compacta can be described by some nonnegative functions $\psi$ satisfying condition (B) (or condition (B1), when decompositions are considered) such that a space $X$ has the property if and only if $\psi(X)=0$. We have already mentioned one such property: the hereditary indecomposability represented by the function $\xi$. Other examples are: dimension less than $n$, arc-likeness, tree-likeness, $P$-likeness (where $P$ is a class of polyhedra), 
atriodicity, hereditary unicoherence, etc. Normally, properties of compacta preserved by inverse limits have such representations, and they are a natural domain for applications of the results of this section. In the next section, proving the main result of the paper, we give an example of such an application.

Main result. Let $\mathcal{S}$ be the unit circle $\left\{(x, y) \in \mathbb{R}^{2}: x^{2}+y^{2}=1\right\}$ with the Euclidean metric $d$, and $\mathcal{A}$ be the annulus $[0,1] \times \mathcal{S}$. Define the set $\mathcal{A}_{0}=$ $\{0,1\} \times \mathcal{S}$ and the family $\mathcal{M}_{\mathcal{A}}$ of all surjective mappings $f: \mathcal{A} \rightarrow \mathcal{A}$ such that $f(p)=p$ for each $p \in \mathcal{A}_{0}$, and observe that $\mathcal{M}_{\mathcal{A}}$ satisfies condition (A).

The following lemma was shown in [9] (Theorem 3). Actually, it was proved for the product $\mathbb{R} \times \mathcal{S}$, and $\varepsilon$-hereditary indecomposability is obtained for the intersections of the fibers with a given bounded subset of $\mathbb{R} \times \mathcal{S}$. However, taking $[0,1]$ for the compactification of $\mathbb{R}$, the set $U$ there sufficiently large and $\varepsilon$ sufficiently small, the lemma below follows. A related idea is also implicit in Lemmas 5.2 and 5.3 of [6]. The symbol $\pi$ denotes the projection of $\mathcal{A}$ onto its second coordinate.

Lemma 6. For any $\varepsilon>0$ there exists a homeomorphism $h \in \mathcal{M}_{\mathcal{A}}$ such that:

(a) the mapping $f_{h}: \mathcal{A} \rightarrow \mathcal{A}$ defined by $f_{h}\left(x_{0}, x_{1}\right)=\left(x_{0}, \pi\left(h^{-1}\left(x_{0}, x_{1}\right)\right)\right)$ is an $\varepsilon$-pushing, and

(b) $\xi(h([0,1] \times\{x\}))<\varepsilon$ for each $x \in \mathcal{S}$.

We say that two decompositions of the same space are topologically equivalent provided that there is an autohomeomorphism of the space transforming one decomposition onto the other.

For any two continuous decompositions $\mathcal{D}_{1}, \mathcal{D}_{2}$ of $\mathcal{A}$ define $\sigma\left(\mathcal{D}_{1}, \mathcal{D}_{2}\right)$ according to the definition (1), where $\Phi=\mathcal{M}_{\mathcal{A}}$.

Let $\Gamma_{0}$ be the family of all decompositions $\mathcal{D}$ of $\mathcal{A}$ topologically equivalent to the decomposition $\mathcal{D}_{0}=\{[0,1] \times\{x\}: x \in \mathcal{S}\}$ such that there exists a homeomorphism in $\mathcal{M}_{\mathcal{A}}$ transforming $\mathcal{D}$ onto $\mathcal{D}_{0}$.

Define $\Gamma$ as the set of all continuous decompositions $\mathcal{D}$ of $\mathcal{A}$ into arc-like continua such that each element $D$ of $\mathcal{D}$ intersects $\mathcal{A}_{0}$ at exactly two points that have the same second coordinate, and $D$ is irreducible between these two points. We have $\Gamma_{0} \subset \Gamma$.

Observe that the set $\Gamma$ is closed with respect to the invlim $_{\sigma}$ convergence. Indeed, assume $\mathcal{D}=\operatorname{invlim}_{\sigma} \mathcal{D}_{n}$, where $\mathcal{D}$ is an upper semicontinuous decomposition of $\mathcal{A}$ into compacta and $\mathcal{D}_{n} \in \Gamma$. Let $f_{n}: \mathcal{A} \rightarrow \mathcal{A}$ be the mappings in $\mathcal{M}_{\mathcal{A}}$ guaranteed by the definition. Since $\varepsilon$-pushings that transform one upper semicontinuous decomposition onto another for sufficiently small $\varepsilon$ must preserve discontinuity, we infer that $\mathcal{D}$ is continuous. The mappings $f_{n}$ are identities on $\mathcal{A}_{0}$ and the images of any two different elements of $\mathcal{D}$ 
are disjoint, thus each element of $\mathcal{D}$ intersects $\mathcal{A}_{0}$ at exactly two points having the same second coordinate. The mapping $f_{n}$ transforms an element $D$ of $\mathcal{D}$ onto an element $D_{n}$ of $\mathcal{D}_{n}$ which is irreducible between the points of intersection with $\mathcal{A}_{0}$ for each $n$. So $D$ has the same property. Since the $D_{n}$ 's are chainable, so is $D$.

Next, for any decomposition $\mathcal{D} \in \Gamma$ define the number $\xi(\mathcal{D})=\sup \{\xi(D)$ : $D \in \mathcal{D}\}$. This number measures the decomposability of subcontinua of the elements of $\mathcal{D}$. All elements of $\mathcal{D}$ are hereditarily indecomposable if and only if $\xi(\mathcal{D})=0$.

Note that the mapping $f_{h}$ in Lemma 6 transforms the decomposition $\{h([0,1] \times\{x\}): x \in \mathcal{S}\}$ onto the decomposition $\mathcal{D}_{0}$. Thus Lemma 6 implies the following one, which proves property $(\mathrm{C} 1)$ for $\xi$ in $\Gamma_{0}$.

Lemma 7. For each $\mathcal{D} \in \Gamma_{0}$ there exists a sequence $\mathcal{D}_{n}$ in $\Gamma_{0}$ such that $\mathcal{D}=\lim _{\sigma} \mathcal{D}_{n}$ and $\lim \xi\left(\mathcal{D}_{n}\right)=0$.

By Example 2 the function $\xi$ on $\Gamma$ satisfies condition (B1).

Applying Theorem 5 for the above-defined $\sigma$ for the families $\Gamma$ and $\Gamma_{0}$ we obtain the following theorem, which is a reformulation of the main result of [9] (Theorem 7).

TheOREm 8. For each $\mathcal{D} \in \Gamma_{0}$ there is a sequence $\mathcal{D}_{n}$ in $\Gamma$ such that $\mathcal{D}=\lim _{\sigma} \mathcal{D}_{n}$ and $\xi\left(\mathcal{D}_{n}\right)=0$ for each $n$.

For any $K \in C(\mathcal{A})$ define $\alpha(K)=\inf \left\{d(x, y): x \in K\right.$ and $\left.y \in \mathcal{A}_{0}\right\}$. Let $\mathcal{D} \in \Gamma, p \in \mathcal{A}, I(p, \mathcal{D})$ be the element of $\mathcal{D}$ containing $p$, and $(0, x),(1, x)$ be the two points of the intersection $I(p, \mathcal{D}) \cap \mathcal{A}_{0}$. The function $\pi_{\mathcal{D}}(p)=x$ is an open monotone mapping onto $\mathcal{S}$. Let $I_{i}(p, \mathcal{D})$ be the unique continuum in $I(\mathcal{D}, p)$ irreducible between $p$ and $(i, x)$ for $i \in\{0,1\}$. For any nonempty set $A \subset \mathcal{A}$ define $\delta_{\mathcal{D}}(A)=\operatorname{diam} \pi_{\mathcal{D}}(A)$.

Fix a Whitney mapping $w: C(\mathcal{A}) \rightarrow[0, \infty)$. For any $\mathcal{D} \in \Gamma$ define the function $\gamma_{\mathcal{D}}: \mathcal{A} \rightarrow \mathbb{R}$ by

$$
\gamma_{\mathcal{D}}(p)=w(I(p, \mathcal{D}))-\frac{w\left(I_{0}(p, \mathcal{D})\right)+w\left(I_{1}(p, \mathcal{D})\right)}{2} .
$$

Observe that $\gamma_{\mathcal{D}}$ is nonnegative, and

$$
\begin{aligned}
& \lim \gamma_{\mathcal{D}}\left(p_{n}\right)=0 \text { if and only if } \lim \left(w\left(I\left(p_{n}, \mathcal{D}\right)\right)-w\left(I_{0}\left(p_{n}, \mathcal{D}\right)\right)\right)= \\
& \lim \left(w\left(I\left(p_{n}, \mathcal{D}\right)\right)-w\left(I_{1}\left(p_{n}, \mathcal{D}\right)\right)\right)=0 .
\end{aligned}
$$

For any $K \in C(\mathcal{A})$ define $\gamma_{\mathcal{D}}(K)=\inf \left\{\gamma_{\mathcal{D}}(p): p \in K\right\}$. Finally let

$$
\begin{aligned}
\beta_{\mathcal{D}}(K) & =\min \left\{\alpha(K), \delta_{\mathcal{D}}(K), \gamma_{\mathcal{D}}(K)\right\} \quad \text { for } K \in C(\mathcal{A}), \\
\beta(\mathcal{D}) & =\sup \left\{\beta_{\mathcal{D}}(K): K \in C(\mathcal{A})\right\} .
\end{aligned}
$$

We will prove that the function $\beta$, similarly to $\xi$ (see Lemma 7 ), satisfies condition $(\mathrm{C} 1)$ for $\Gamma_{0}$. 
Lemma 9. For each $\mathcal{D} \in \Gamma_{0}$ there exists a sequence $\mathcal{D}_{n}$ in $\Gamma_{0}$ such that $\mathcal{D}=\lim _{\sigma} \mathcal{D}_{n}$ and $\lim \beta\left(\mathcal{D}_{n}\right)=0$.

Proof. It suffices to prove the lemma for $\mathcal{D}=\mathcal{D}_{0}$ (all other elements of $\Gamma_{0}$ are topologically equivalent to $\left.\mathcal{D}_{0}\right)$.

Take the decompositions $\mathcal{D}_{n}$ guaranteed by Lemma 7 for $\mathcal{D}_{0}$. Suppose this sequence does not have the required property. Then there is an $\varepsilon \in$ $(0,1 / 2)$ and a sequence $\left\{K_{n}\right\} \subset C(\mathcal{A})$ such that $\beta_{\mathcal{D}_{n}}\left(K_{n}\right)>\varepsilon$ for each $n$. We observe that the mappings $\pi_{\mathcal{D}_{n}}$ uniformly converge to the projection $\pi$ onto the second coordinate $\mathcal{S}$. Thus diam $\pi\left(K_{n}\right)>\varepsilon / 2$ for almost all $n$ (assume this inequality holds for all $n$ ). For any $n$ take a continuum $L_{n} \subset K_{n}$ such that $\operatorname{diam} \pi\left(L_{n}\right)=\varepsilon / 2$. Taking a subsequence, we assume that $\operatorname{Lim} L_{n}=L$ for some continuum $L \in C(\mathcal{A})$. Thus $\pi\left(L_{n}\right)$ is a sequence of arcs in $\mathcal{S}$ converging to an arc $x y$ in $\mathcal{S}$ with $d(x, y)=\varepsilon / 2$. Let $z$ be the midpoint of the arc $x y$ and $A_{n}=\pi_{\mathcal{D}_{n}}^{-1}(z)$. By Lemma 7 we have $\lim \xi\left(A_{n}\right)=0$. Therefore there are two different points $r_{n}, s_{n} \in A_{n} \backslash\{(0, z),(1, z)\}$ such that the ordering $(0, z), r_{n}, s_{n},(1, z)$ agrees with the ordering of $A_{n}$, and $\lim d\left(r_{n},(1, z)\right)=$ $\lim d\left(s_{n},(0, z)\right)=0$. Since $\alpha\left(L_{n}\right) \geq \alpha\left(K_{n}\right)>\varepsilon$, the arc $r_{n} s_{n} \subset A_{n}$ must intersect $L_{n}$ for almost all $n$. Let $B_{n}, C_{n}$ be arcs in $A_{n}$ irreducible between $(0, z), r_{n}$, and between $s_{n},(1, z)$, respectively. We have $\operatorname{Lim} A_{n}=\operatorname{Lim} B_{n}=$ $\operatorname{Lim} C_{n}=\operatorname{Lim} r_{n} s_{n}$. This implies that $\lim _{n}\left(\sup \left\{\gamma_{\mathcal{D}_{n}}(p): p \in r_{n} s_{n}\right\}\right)=0$. So $\lim \gamma_{\mathcal{D}_{n}}\left(L_{n}\right)=0$, and thus $\lim \gamma_{\mathcal{D}_{n}}\left(K_{n}\right)=0$. Hence $\lim \beta_{\mathcal{D}_{n}}\left(K_{n}\right)=0$, a contradiction.

We prove that the function $\beta$ satisfies condition (B1).

LemMa 10. For any sequence $\mathcal{D}, \mathcal{D}_{1}, \mathcal{D}_{2}, \ldots$ in $\Gamma$ such that $\operatorname{invlim}_{\sigma} \mathcal{D}_{n}$ $=\mathcal{D}$ we have $\beta(\mathcal{D}) \leq \liminf \beta\left(\mathcal{D}_{n}\right)$.

Proof. Assume that $\beta(\mathcal{D})>\varepsilon$. Then there is a $K \in C(\mathcal{A})$ with $\beta_{\mathcal{D}}(K)>\varepsilon$. Let $f_{n}: \mathcal{A} \rightarrow \mathcal{A}$ be mappings in $\Gamma$ transforming $\mathcal{D}$ onto $\mathcal{D}_{n}$ with $\lim d\left(f_{n}\right)=0$, and let $K_{n}=f_{n}(K)$. We have $\lim \inf \alpha\left(K_{n}\right)>\varepsilon$. We also observe that the projections $\pi_{\mathcal{D}_{n}}$ uniformly converge to the projection $\pi_{\mathcal{D}}$, and thus $\liminf \delta_{\mathcal{D}_{n}}\left(K_{n}\right)>\varepsilon$. Note that the function $w\left(I\left(f_{n}(p), \mathcal{D}_{n}\right)\right)$ uniformly converges to $w(I(p, \mathcal{D}))$ on $K$. We also have

$$
\lim \sup w\left(I_{i}\left(f_{n}(p), \mathcal{D}_{n}\right)\right) \leq w\left(I_{i}(p, \mathcal{D})\right)
$$

for each $p \in K$ and $i \in\{0,1\}$. Therefore $\liminf \gamma_{\mathcal{D}_{n}}\left(f_{n}(p)\right) \geq \gamma_{\mathcal{D}}(p)$. Moreover, the estimate (4) for the sequences $\sup \left\{w\left(I_{i}\left(f_{n}(p), \mathcal{D}_{n}\right)\right): n>m\right\}$ is uniform (it depends on $m$ only - does not depend on $p$ ) by the uniform convergence of the mappings $f_{n}$. Thus we also have

$$
\liminf \gamma_{\mathcal{D}_{n}}\left(K_{n}\right) \geq \gamma_{\mathcal{D}}(K)>\varepsilon
$$

Hence $\lim \inf \beta\left(\mathcal{D}_{n}\right)>\varepsilon$. The proof is complete. 
Let $\mathcal{P}$ be the infinite product $[0,1] \times \mathcal{S}^{\omega}$. Define $\mathcal{P}_{0}=\{0,1\} \times \mathcal{S}^{\omega}$, and consider the family $\mathcal{M}_{\mathcal{P}}$ of all surjective mappings $f: \mathcal{P} \rightarrow \mathcal{P}$ such that $f(p)=p$ for each $p \in \mathcal{P}_{0}$. Observe that $\mathcal{M}_{\mathcal{P}}$ satisfies condition $(\mathrm{A})$.

For $\mathcal{P}$ we define similar concepts to those defined for the annulus $\mathcal{A}$. In some cases we keep the same symbols as those used for $\mathcal{A}$.

For any two continuous decompositions $\mathcal{D}_{1}, \mathcal{D}_{2}$ of $\mathcal{P}$ define $\sigma\left(\mathcal{D}_{1}, \mathcal{D}_{2}\right)$ according to the definition (1), where $\Phi=\mathcal{M}_{\mathcal{P}}$.

Let $\Omega_{0}$ be the family of all decompositions of $\mathcal{P}$ topologically equivalent to the decomposition $\mathcal{D}_{0}=\left\{[0,1] \times\{x\}: x \in \mathcal{S}^{\omega}\right\}$, such that the homeomorphism defining this equivalence belongs to $\mathcal{M}_{\mathcal{P}}$.

Define $\Omega$ as the set of all continuous decompositions $\mathcal{D}$ of $\mathcal{P}$ into arc-like continua such that each element $D$ of $\mathcal{D}$ intersects $\mathcal{P}_{0}$ at exactly two points which have all but the first coordinate the same, and $D$ is irreducible between these two points. We have $\Omega_{0} \subset \Omega$.

For any $K \in C(\mathcal{P})$ define $\alpha(K)=\inf \left\{d(x, y): x \in K\right.$ and $\left.y \in \mathcal{P}_{0}\right\}$. Let $\mathcal{D} \in \Omega, p \in \mathcal{P}, I(p, \mathcal{D})$ be the element of $\mathcal{D}$ containing $p$, and $(0, s),(1, s)$ be the two points of the intersection $I(p, \mathcal{D}) \cap \mathcal{P}_{0}$, where $s=\left(s_{1}, s_{2}, \ldots\right) \in \mathcal{S}^{\omega}$. The function $\pi_{n, \mathcal{D}}(p)=s_{n}$ is an open monotone mapping onto $\mathcal{S}$. For any nonempty set $A \subset \mathcal{P}$ and any $n \in\{1,2, \ldots\}$ define $\delta_{n, \mathcal{D}}(A)=\operatorname{diam} \pi_{n, \mathcal{D}}(A)$. Let $I_{i}(p, \mathcal{D})$ be the unique continuum in $I(\mathcal{D}, p)$ irreducible between $p$ and $(i, s)$ for $i \in\{0,1\}$.

Fix a Whitney mapping $w: C(\mathcal{P}) \rightarrow[0, \infty)$. For any $\mathcal{D} \in \Omega$ define the function $\gamma_{\mathcal{D}}: \mathcal{P} \rightarrow \mathbb{R}$ by $(2)$. Observe that $\gamma_{\mathcal{D}}$ is nonnegative and it satisfies condition (3).

For any $K \in C(\mathcal{P})$ define $\gamma_{\mathcal{D}}(K)=\inf \left\{\gamma_{\mathcal{D}}(p): p \in K\right\}$. Finally, let

$$
\begin{aligned}
\beta_{n, \mathcal{D}}(K) & =\min \left\{\alpha(K), \delta_{n, \mathcal{D}}(K), \gamma_{\mathcal{D}}(K)\right\} \quad \text { for } K \in C(\mathcal{P}), \\
\beta_{n}(\mathcal{D}) & =\sup \left\{\beta_{n, \mathcal{D}}(K): K \in C(\mathcal{P})\right\} .
\end{aligned}
$$

Applying Lemma 7 for the two initial coordinates of $\mathcal{P}$, we have the following lemma, which states property (C1) for the function $\xi$ in $\Omega_{0}$.

Lemma 11. For each $\mathcal{D} \in \Omega_{0}$ there exists a sequence $\mathcal{D}_{n}$ in $\Omega_{0}$ such that $\mathcal{D}=\lim _{\sigma} \mathcal{D}_{n}$ and $\lim \xi\left(\mathcal{D}_{n}\right)=0$.

The next two lemmas formulate properties (C1) and (B1) for the functions $\beta_{n}$ in $\Omega_{0}$ and $\Omega$, respectively. Their proofs easily follow from the corresponding ones of Lemmas 9 and 10. We present a construction that leads to the proof of Lemma 12. The proof of Lemma 13 is left to the reader.

Lemma 12. For each $\mathcal{D} \in \Omega_{0}$ and for each $n \in\{1,2, \ldots\}$ there exists a sequence $\mathcal{D}_{m}$ in $\Omega_{0}$ such that $\lim _{\sigma} \mathcal{D}_{m}=\mathcal{D}$ and $\lim _{m \rightarrow \infty} \beta_{n}\left(\mathcal{D}_{m}\right)=0$. 
Proof. First we observe that the mapping

$$
p \mapsto\left(\frac{w\left(I_{0}(p, \mathcal{D})\right)}{w(I(p, \mathcal{D}))}, \pi_{1, \mathcal{D}}(p), \pi_{2, \mathcal{D}}(p), \ldots\right)
$$

defines a homeomorphism $h: \mathcal{P} \rightarrow \mathcal{P}$ belonging to $\mathcal{M}_{\mathcal{P}}$. Let $\mathcal{D}_{m}^{\prime}$ be the decompositions of $\mathcal{A}$ guaranteed by Lemma 9 for the decomposition $\mathcal{D}_{0}$. Fix an integer $m$. In $\mathcal{P}$ we define a decomposition $\mathcal{D}_{m}^{\prime \prime}$ such that each element $D^{\prime \prime}$ of $\mathcal{D}_{m}^{\prime \prime}$ is composed of the points having the first and the $(n+1)$ st coordinates identical to the coordinates of the points of some element $D^{\prime}$ of $\mathcal{D}_{m}^{\prime}$ while the other coordinates of the points of $D^{\prime \prime}$ are constant. Finally, we define $\mathcal{D}_{m}=\left\{h^{-1}\left(D^{\prime \prime}\right): D^{\prime \prime} \in \mathcal{D}_{m}^{\prime \prime}\right\}$. The decompositions $\mathcal{D}_{m}$ satisfy the conclusion of the lemma.

Lemma 13. For any sequence $\mathcal{D}, \mathcal{D}_{1}, \mathcal{D}_{2}, \ldots$ in $\Omega$ such that $\operatorname{invlim}_{\sigma} \mathcal{D}_{m}$ $=\mathcal{D}$ and any $n \in\{1,2, \ldots\}$ we have $\beta_{n}(\mathcal{D}) \leq \liminf \beta_{n}\left(\mathcal{D}_{m}\right)$.

Now we apply Theorem 5 for the functions $\psi_{1}=\xi, \psi_{2}=\beta_{1}, \psi_{3}=\beta_{2}, \ldots$ to obtain the main result of this paper.

Corollary 14. For any $\mathcal{D} \in \Omega_{0}$ there exists a sequence $\mathcal{D}_{m}$ of decompositions in $\Omega$ such that $\lim _{\sigma} \mathcal{D}_{m}=\mathcal{D}$ and $0=\xi\left(\mathcal{D}_{m}\right)=\beta_{1}\left(\mathcal{D}_{m}\right)=\beta_{2}\left(\mathcal{D}_{m}\right)$ $=\ldots$ for each $m \in\{1,2, \ldots\}$.

Let $\mathcal{D}_{*}$ be one of the decompositions $\mathcal{D}_{m}$ guaranteed by Corollary 14 . Since $\mathcal{D}_{*} \in \Omega$, the elements of $\mathcal{D}_{*}$ are arc-like, and the set $\mathcal{P}_{0}^{\prime}=\{0\} \times \mathcal{S}^{\omega}$ is a continuous selector of this decomposition. Thus the quotient space of this decomposition is homeomorphic to $\mathcal{S}^{\omega}$. The elements of $\mathcal{D}_{*}$ are hereditarily indecomposable $\left(\xi\left(\mathcal{D}_{*}\right)=0\right)$, so these elements are pseudo-arcs [2].

Let $K \in C\left(\mathcal{P} \backslash \mathcal{P}_{0}\right)$ be a continuum that intersects two different elements of $\mathcal{D}_{*}$. We have $\beta_{n, \mathcal{D}_{*}}(K)=0$, and $\alpha(K), \delta_{n, \mathcal{D}_{*}}(K)>0$ for some $n$. So $\gamma_{\mathcal{D}_{*}}(K)=0$. By the definition of $\gamma_{\mathcal{D}_{*}}(K)$ the continuum $K$ contains a sequence of points $p_{m}$ such that $\lim \left(w\left(I\left(p_{m}, \mathcal{D}_{*}\right)\right)-w\left(I_{0}\left(p_{m}, \mathcal{D}_{*}\right)\right)\right)=$ $\lim \left(w\left(I\left(p_{m}, \mathcal{D}_{*}\right)\right)-w\left(I_{1}\left(p_{m}, \mathcal{D}_{*}\right)\right)\right)=0$. Thus we have the following special property of $\mathcal{D}_{*}$.

Property 15. If a continuum $K$ in $\mathcal{P} \backslash \mathcal{P}_{0}$ intersects two different elements of $\mathcal{D}_{*}$, then there is a sequence $\left\{p_{m}\right\} \subset K$ such that $\lim \left(w\left(I\left(p_{m}, \mathcal{D}_{*}\right)\right)\right.$ $\left.-w\left(I_{0}\left(p_{m}, \mathcal{D}_{*}\right)\right)\right)=\lim \left(w\left(I\left(p_{m}, \mathcal{D}_{*}\right)\right)-w\left(I_{1}\left(p_{m}, \mathcal{D}_{*}\right)\right)\right)=0$.

To obtain the announced pseudo-hairy spaces, fix an $a>0$ such that $a<\inf \left\{w(D): D \in \mathcal{D}_{*}\right\}$ and take the family

$$
\mathcal{D}_{a}=\left\{D \in C(\mathcal{P}): D \subset D_{*} \text { for some } D_{*} \in \mathcal{D}_{*} \text {, and } w(D)=a\right\} .
$$

As in [9] (after Th. 7) we prove that $\mathcal{D}_{a}$ is a continuous decomposition of $\mathcal{P}$ into pseudo-arcs. Define the compactum

$$
\mathcal{H}_{a}=\bigcup\left\{D \in \mathcal{D}_{a}: D \cap \mathcal{P}_{0}^{\prime} \neq \emptyset\right\} .
$$


By the continuity of $\mathcal{D}_{a}$ the elements of $\mathcal{D}_{a}$ contained in $\mathcal{H}_{a}$ form a continuous decomposition of $\mathcal{H}_{a}$ into pseudo-arcs. The set $\mathcal{P}_{0}^{\prime}$ is a continuous selector for this decomposition. Let $r: \mathcal{H}_{a} \rightarrow \mathcal{P}_{0}^{\prime}$ be the retraction induced by this selection. Thus the quotient space is homeomorphic to $\mathcal{S}^{\omega}$. For any $p \in \mathcal{H}_{a}$ we have $w\left(I\left(p, \mathcal{D}_{*}\right)\right)-w\left(I_{0}\left(p, \mathcal{D}_{*}\right)\right) \geq \inf \left\{w(D): D \in \mathcal{D}_{*}\right\}-a>0$. Therefore any continuum in $\mathcal{H}_{a}$ joining two different elements of $\mathcal{D}_{a}$ must intersect $\mathcal{P}_{0}^{\prime}$ by Property 15 . Hence $\mathcal{H}_{a}$ is a continuous pseudo-hairy space over the set $\mathcal{P}_{0}^{\prime}$.

Now we are ready to formulate the main result of the paper.

TheOREm 16. For any nonempty compact metric space $X$ there exist a continuous pseudo-hairy space over $X$ and a continuous pseudo-fan of $X$.

Proof. Since the product $\mathcal{S}^{\omega}$, as well as the Hilbert cube, is a universal separable metric space, we may assume that $X$ is contained in $\mathcal{P}_{0}^{\prime}=\{0\} \times \mathcal{S}^{\omega}$. Take $r^{-1}(X)$ for $\widetilde{X}$. The mapping $r$ restricted to $\tilde{X}$ induces a continuous pseudo-hairy structure on $\tilde{X}$. Finally, the quotient space $\tilde{X} / X$, obtained by shrinking $X$ to a point, is the required continuous pseudo-fan of $X$.

REMARK 2. We can naturally generalize the notions of a continuous pseudo-hairy space $\widetilde{X}$ and of a continuous pseudo-fan of $X$ to any separable metric space $X$ (assuming that $r^{-1}(K)$ is compact if $K \subset X$ is compact). Then Theorem 16 remains valid for all separable metric spaces. The proof is similar.

\section{Basic observations on pseudo-hairy spaces and pseudo-fans.} Now, we collect a number of observations on the classes of spaces introduced in this paper. Some of them deal with all pseudo-hairy spaces and all pseudo-fans (not necessarily continuous).

First, we record the following obvious facts. We omit the proof.

Proposition 17. (a) For any connected pseudo-hairy space $\tilde{X}$ different from the pseudo-arc, its base $X$ and the retraction $r$ inducing the pseudohairy structure of $\widetilde{X}$ are uniquely determined.

(b) For any pseudo-fan $Y$ different from the pseudo-arc, its vertex $c$ and the family $\mathcal{F}$ of pseudo-arcs inducing the pseudo-fan structure of $Y$ are uniquely determined. Moreover, if $Y$ is a continuous pseudo-fan of a space $X$, then $X$ is topologically unique.

Next, we prove the following proposition which says that pseudo-fans are star-like (thus tree-like) curves.

Proposition 18. For any pseudo-fan $Y$ and for any $\varepsilon>0$ there exists an $\varepsilon$-star cover $\mathcal{C}$ of $Y$ such that the vertex of $Y$ belongs to the ramification link of $\mathcal{C}$. 
Proof. Let $Y$ be a pseudo-fan with vertex $c$, and let $\mathcal{F}$ be the family of pseudo-arcs inducing the pseudo-fan structure of $Y$. First, we will show that

for any $\delta>0$ and any $P \in \mathcal{F}$ there exists a $\delta$-chain $\mathcal{B}_{P}=\left\{B_{1}, \ldots, B_{k}\right\}$ of open sets in $Y$ such that $P \subset B^{*}=B_{1} \cup \ldots \cup B_{k}$, bd $B^{*} \subset B_{1}$ and $c \in B_{1} \backslash B_{2}$.

Indeed, take a $\delta$-chain $A_{1}, \ldots, A_{k}$ of open sets in $Y$ such that $c \in A_{1} \backslash \operatorname{cl} A_{2}$ and $P \subset A^{*}=A_{1} \cup \ldots \cup A_{k}$, and take an open neighborhood $G$ of $c$ with $\operatorname{cl} G \subset A_{1} \backslash \operatorname{cl} A_{2}$. Since each nondegenerate subcontinuum of $Y \backslash G$ is a pseudo-arc and $P$ is a maximal pseudo-arc in $Y$, each component $K$ of $P \backslash G$ is a component of $Y \backslash G$. Thus such a $K$ has a neighborhood $U_{K} \subset A^{*}$ satisfying bd $U_{K} \subset A_{1} \backslash \operatorname{cl} A_{2}$. The family $\left\{U_{K}\right\}$, for all components $K$ of $P \backslash G$, is an open covering of $P \backslash G$ in $Y$. By the compactness of $P \backslash G$ there are components $K_{1}, \ldots, K_{n}$ of $P \backslash G$ such that $P \backslash G \subset U^{*}=$ $U_{K_{1}} \cup \ldots \cup U_{K_{n}}$ and bd $U^{*} \subset A_{1} \backslash \operatorname{cl} A_{2}$. We take $B_{1}=G \cup\left(A_{1} \cap \operatorname{cl} U^{*}\right)$ and $B_{i}=A_{i} \cap U^{*}$ for $i \in\{2, \ldots, k\}$. These sets have the properties required in (5).

Fix any $\varepsilon>0$. For any $P \in \mathcal{F}$ take an $(\varepsilon / 3)$-chain open covering $\mathcal{B}_{P}$ with the two initial links $B_{0, P}, B_{1, P}$ and the union $B_{P}^{*}$ satisfying (5). By the compactness of $Y$ there is a finite sequence $P_{1}, \ldots, P_{l} \in \mathcal{F}$ such that $B_{P_{1}}^{*} \cup \ldots \cup B_{P_{l}}^{*}=Y$.

Finally, we modify these chain coverings to obtain the required star cover. Namely, we take $C_{0}=\bigcup_{i=1}^{l}\left(B_{0, P_{i}} \cup B_{1, P_{i}}\right)$, and the finite family $\left\{\mathcal{C}_{i}: i \in\{1, \ldots, l\}\right\}$ defined as follows: we let $V_{i}=B_{P_{i}}^{*} \backslash \operatorname{cl} B_{0, P_{i}}$ for $i \in\{1, \ldots, l\}$ and

$$
\mathcal{C}_{i}=\left\{B \backslash\left(V_{1} \cup \ldots \cup V_{i-1}\right): B \in \mathcal{B}_{P_{i}} \text { and } B_{0, P_{i}} \neq B \neq B_{1, P_{i}}\right\} .
$$

The reader can verify that the family $\left\{C_{0}\right\} \cup \mathcal{C}_{1} \cup \ldots \cup \mathcal{C}_{l}$ is the required $\varepsilon$-star cover of $Y$.

Observe that the above proposition implies that each pseudo-fan is an inverse limit of $n$-ods (for varying $n$ ) with bonding maps preserving the ramification point. In particular, pseudo-fans are tree-like and 1-dimensional, so they are embeddable in $\mathbb{R}^{3}$. Thus such "simple" spaces keep the whole information on all separable metric spaces (comp. Proposition 17).

Among these spaces there appear a continuous pseudo-fan of the Hilbert cube $Y_{H}$ such that for any compactum $X$ there is a continuous pseudo-fan $Y_{X}$ of $X$ contained in $Y_{H}$. Observe that the pseudo-fans $Y_{H}$ and $Y_{X}$ have the same vertex, and each pseudo-arc in $Y_{X}$ is contained in some pseudo-arc from the pseudo-fan structure of $Y_{H}$.

We record some dimension properties of pseudo-hairy spaces and pseudofans. 
Proposition 19. For any pseudo-hairy space $\widetilde{X}$ over a compact space $X$ and for any pseudo-fan $Y_{X}$ of $X$ we have $\operatorname{dim}(\tilde{X}-X)=\operatorname{dim} Y_{X}=1$ and $\operatorname{dim} \tilde{X}=\max \{1, \operatorname{dim} X\}$.

In fact, the space $\widetilde{X}-X$ is locally compact and has only pseudo-arcs for its nondegenerate subcontinua, and $Y_{X}$ is the one-point compactification of $\widetilde{X}-X$, thus the conclusion follows.

TheOREM 20. For any compact space $X$ embeddable in $\mathbb{R}^{n}$, there exists a continuous pseudo-hairy space $\widetilde{X}$ over $X$ embeddable in $\mathbb{R}^{n+1}$.

Indeed, we embed $X$ in the product $[0,1]^{n}$. Next, we embed this product in $\mathcal{S}^{n}$ and repeat the main construction for $[0,1] \times \mathcal{S}^{n}$ instead of $[0,1] \times \mathcal{S}^{\omega}$.

The following result was obtained in [9] (Ths. 8 and 16).

THEOREM 21. There exists a plane continuous pseudo-hairy space $\tilde{X}$ over a nondegenerate continuum $X$ if and only if $X$ is either an arc, or a simple closed curve.

W. Lewis proved in [7] the following property of the pseudo-arc: for any sequence $P_{n}$ of pseudo-arcs converging to a pseudo-arc $P$ (in the sense of the Hausdorff distance) there are homeomorphisms $h_{n}: P_{n} \rightarrow P$ such that $\lim d\left(h_{n}\right)=0$. Let $p_{n} \in P_{n}$ and $p \in P$ with $\lim p_{n}=p$. By the homogeneity of the pseudo-arc, and by the Effros' theorem, we can take the homeomorphisms $h_{n}$ additionally satisfying $h_{n}\left(p_{n}\right)=p$. This implies the following homeomorphic continuity of the pseudo-hairy structure of continuous pseudo-hairy spaces and continuous pseudo-fans.

TheOrem 22. (a) Let $\widetilde{X}$ be a continuous pseudo-hairy space over a compactum $X$ with the retraction $r: \widetilde{X} \rightarrow X$ inducing the pseudo-hairy structure of $\widetilde{X}$. For each $\varepsilon>0$ there exists a $\delta>0$ such that for each pair of points $p, q \in \widetilde{X}$ with $d(p, q)<\delta$ there exists a homeomorphism $h: r^{-1}(r(p)) \rightarrow r^{-1}(r(q))$ which is an E-pushing such that $h(p)=q$.

(b) Let $Y$ be a continuous pseudo-fan with the family $\mathcal{F}$ of pseudo-arcs inducing the pseudo-fan structure of $Y$. For each $\varepsilon>0$ there exists a $\delta>0$ such that for each pair of points $p, q \in Y$, if $p \in P \in \mathcal{F}, q \in Q \in \mathcal{F}$ and $d(p, q)<\delta$, then there exists a homeomorphism $h: P \rightarrow Q$ which is an $\varepsilon$-pushing such that $h(p)=q$.

We end the paper with the following natural question. (It is known that the answer is affirmative if $X$ is 0-dimensional.)

Question 2. Given a compactum $X$, is the continuous pseudo-hairy space over $X$ topologically unique? Is the continuous pseudo-fan of $X$ topologically unique? 


\section{References}

[1] R. D. Anderson and G. Choquet, A plane continuum no two of whose nondegenerate subcontinua are homeomorphic: an application of inverse limits, Proc. Amer. Math. Soc. 10 (1959), 347-353.

[2] R. H. Bing, Concerning hereditarily indecomposable continua, Pacific J. Math. 1 (1951), 43-51.

[3] R. H. Bing and F. B. Jones, Another homogeneous plane continuum, Trans. Amer. Math. Soc. 90 (1959), 171-192.

[4] R. J. Daverman, Decompositions of Manifolds, Academic Press, 1986.

[5] B. Knaster, Un continu dont tout sous-continu est indécomposable, Fund. Math. 3 (1922), 247-286.

[6] J. Krasinkiewicz, On mappings with hereditarily indecomposable fibers, Bull. Polish Acad. Sci. Math. 44 (1996), 147-156.

[7] W. Lewis, Observations on the pseudo-arc, Topology Proc. 9 (1984), 329-337.

[8] —, Continuous curves of pseudo-arcs, Houston J. Math. 11 (1985), 91-99.

[9] J. R. Prajs, A continuous circle of pseudo-arcs filling up the annulus, Trans. Amer. Math. Soc. 352 (2000), 1743-1757.

Institute of Mathematics

Opole University

Oleska 48

45-052 Opole, Poland
Current address:

Department of Mathematics

Idaho State University

Pocatello, ID 83209, U.S.A.

E-mail: prajs@math.isu.edu

Received 16 August 1999;

in revised form 12 June 2001 